\title{
Análisis del fenómeno del TDAH (Trastorno por déficit de atención con hiperactividad) en la Dirección de Psicopedagogía de la Secretaría de Educación Jalisco. Los intentos de hacer invisible la subjetividad en los ambientes escolares
}

DOI: https://doi.org/10.32870/dse.v0i8.306

\section{Talía Chávez Palencia*}

Resumen: El presente trabajo tiene el objetivo de presentar la forma en la que la Dirección de Psicopedagogía perteneciente a la Secretaría de Educación Jalisco, aborda a los alumnos que presentan síntomas relacionados con el Trastorno de Déficit de Atención, como manera de dar una respuesta rápida a la problemática, sin involucrar a las figuras de autoridad. Esto se hace posible gracias a este tipo de diagnósticos que etiquetan a los niños y ponen el acento en una cuestión neurobiológica, dejando de lado los problemas del contexto. Palabras clave: trastorno de déficit de atención, abordaje, etiquetación, dirección de Psicopedagogía, síntomas.

\begin{abstract}
This paper goal is to show the perspective of the Dirección de Psicopedagogía that belongs to the Secretaria de Educación Jalisco, in the treatment with kids that presents ADD. This intervention doesn't involve authority figures. The cause is because diagnosis only see neurobiological facts, and put labels, without taking care of the context's problems where the children grow. Key words: ADD, intervention, labeling, Dirección de Psicopedagogía, symptoms.
\end{abstract}

Es cada vez más común observar las brechas generacionales existentes entre los niños actuales y los actores educativos que los rodean, por eso predominan los comentarios relacionados con lo problemático que resulta ser infante, en esta época, y las conductas extrañas que presentan. Muy pocos adultos que interfieren en la educación de las nuevas generaciones se cuestionan sobre el origen de estos cambios, y usualmente se utilizan los diagnósticos de moda, impuestos por la Asociación Psiquiátrica Americana, a través de su Manual Diagnóstico de Desórdenes Mentales (DSM), para

*Maestra en Estudios Psicoanalíticos Freudianos. Espacio Psicoanalítico, A.C. Psicóloga en la Dirección de Psicopedagogía de la Secretaría de Educación Jalisco. Donato Guerra \#295. Col. Centro. Guadalajara, Jal. 38192727 ext.57763. Correo electrónico: taliach@gmail.com 
dar una rápida respuesta a estas conductas infantiles que confrontan a quienes no entienden cómo abordar estas situaciones; por esta razón, Gabriela Dueñas (2007) comenta que existe una necesidad casi compulsiva de encontrarle un rótulo a cada niño para reubicarlo en el sistema, o dicho en otras palabras, para tratar de proporcionar una explicación a sus conductas. Sin embargo, gran parte de los diagnósticos utilizados sólo encasillan y generan un alivio pasajero a los adultos que buscan respuestas ante los casos correspondientes.

Juan Vasen (2008) en su libro titulado Las Certezas perdidas, retomando los planteamientos de Bauman comenta que las relaciones que se construyen y los lineamientos son líquidos; es decir, que en esta sociedad todo es desechable (hasta las personas), por lo cual es cada vez más frecuente la exclusión escolar, como si se tratara de una selección de alumnos, en la cual los que son considerados como "no funcionales" son expulsados con regularidad, a menos de que puedan cambiar radicalmente su conducta para no ser un estorbo en el aula.

En este sentido, la familia y la escuela han sido identificadas como instituciones fundadoras de marcas que generan lazos sociales; no obstante, pocas veces se toma en cuenta el contexto cultural, en un mundo moderno caracterizado por cambios continuos y bajo preceptos en los que el tiempo implica dinero; además, la vida vertiginosa que llevamos nos impulsa a buscan soluciones rápidas, en lugar de realizar análisis más profundos. A eso debemos sumar el hecho de que los padres ya no son los únicos "modelos a seguir" por parte de sus hijos, pues en la actualidad los personajes de películas y televisión han pasado a ocupar el lugar de estereotipos ante la ausencia de los progenitores, debido a las jornadas largas de trabajo de éstos, o los frágiles lazos que se establecen en los nuevos núcleos familiares.

Hablar de familias disfuncionales como únicas causantes de las problemáticas que enfrentan los niños en la actualidad, es una aseveración que intenta también dar una explicación rápida a su situación, ya que "Las dificultades de aprendizaje de los niños, revelan, en ocasiones que existen agujeros en el tejido de la historia familiar, que es el cimiento de cualquier otro saber" (Colín, 2001: 135).

Uno de los diagnósticos más utilizados es el Trastorno de Déficit de Atención (TDA). No obstante, no proviene esta etiqueta, únicamente de los paidopsiquiatras o neurólogos, sino que actualmente es la escuela y sus actores quienes comienzan a realizar estas hipótesis e intentan que los que pertenecemos al área de la salud, verifiquemos sus premisas. ¿Pero por qué este diagnóstico es cada vez más utilizado en los ambientes educativos? ¿De dónde nace la necesidad de la medicalización de los niños como requisito para poder aprender?

Vasen, J. (2011) refiere que nuestras sociedades están influenciadas por paradigmas tecnocráticos, los cuales tienden a reducir las prácticas sociales complejas como criar, educar, diagnosticar y curar a procedimientos técnicos. Por esta razón, diagnósticos como el Trastorno de Déficit de Atención (TDA), se han ido institucionalizando socialmente cada vez con más popularidad. Su primera aparición, como trastorno, ocurrió en 1980, a partir del DSM-III (Diagnostic and Stadis- 
tical Manual), por la Asociación Norteamericana de Psiquiatría, ya que lo reconoció dentro de su clasificación, otorgándole el sustento necesario para ser encuadrado como una patología.

Sin embargo, el cuestionamiento sobre el discurso de la atención como enfermedad, surgió en 1798 con Alexander Crichton, quien describió un estado mental similar al subtipo de inatención del TDAH. En el capítulo "On attention and its diseases", describió este estado como "mental restlessness". Este último término se puede traducir como: Inquietud, ansiedad, agitación nerviosa, desasosiego, desazón, excitabilidad, excitación, impaciencia, intranquilidad, nerviosismo, sofoquina; insomnio. Desde sus inicios, así como este médico lo planteaba, el mismo término podía significar varias cosas a la vez, y la deficiencia de atención a lo largo de los años ha continuado con estas ambigüedades, que va desde los síntomas físicos que pueden traducirse en trastornos de ansiedad, estrés o depresión, hasta respuestas que se vinculan con las habilidades sociales del sujeto.

Crichton afirmaba que la incapacidad de atender con un necesario grado de constancia hacia cualquier objeto, casi siempre decrecía por una sensibilidad de los nervios, innatural o mórbida, lo cual se traducía en el hecho de que esta facultad incesantemente se retirara de una impresión a otra. Podría nacer en una persona o bien verse afectada por un accidente. Fue el primero que comenzó a poner a discusión si la atención podía ser considerada como una enfermedad.

No obstante, hasta la fecha, aunque los criterios del trastorno se encuentren especificados en el Manual Diagnóstico de la Asociación Psiquiátrica Americana, las conductas descritas abordan situaciones diversas, propias de los procesos de desarrollo en los niños y la valoración de dichas conductas, como son observadas en el ambiente escolar y familiar, pasan a ser evaluadas por las connotaciones morales de las figuras de autoridad, las cuales son las que finalmente emiten juicios acerca de las conductas de los niños.

Ignorando la novela familiar, así como las identificaciones de estos padres y maestros con los sujetos que tienen que examinar ¿qué tan científico u objetivo será este procedimiento? Ese sistema de emitir un diagnóstico en forma rápida, se retroalimenta con mensajes en los que se plantea que los niños con mala conducta o que no siguen las reglas en el aula son fácilmente pronosticados como "futuros delincuentes" o "drogadictos" si no son atendidos con psicofármacos, desde una visión supuestamente médica. Éstas, podrían ser algunas de las razones que más preocupan a los padres, pero ¿será totalmente cierto?

Bange (2010) retoma esta discusión acerca del porvenir de los riesgos que corren los niños que son clasificados con este diagnóstico, y menciona que la experiencia clínica muestra que muchos escapan a estos peligros y se convierten en adultos razonablemente felices. Cita a algunos padres que se reconocen en las dificultades de sus hijos, y descubren haber sacado provecho de ciertas disposiciones asociadas al TDAH: la capacidad de iniciativa y la terquedad, tan frecuentes y a menudo peligrosas en la infancia, a partir de su experiencia, se convirtieron en triunfos una vez conquistada la edad adulta. 
La perspectiva de estos padres de familia, es una forma diferente de abordar estas conductas sin verlas tan catastróficas. Según este punto de vista, se puede abrir el debate acerca de si las conductas a evaluar en este diagnóstico describen la deficiencia o carencia de la atención en diversas actividades, desde una perspectiva neurobiológica, o si pueden ser evaluadas como síntomas. Bajo esta óptica, podrían ser analizadas por la motivación que tienen los niños para realizar ciertas tareas y la relación que en este caso establece el menor con la figura de autoridad que se las solicita. Otro factor es su preparación previa, es decir, si han trabajado con el menor en competencias de organización y planificación de sus tareas, su deseo por estudiar, sus experiencias previas en la escuela, entre otros cuestionamientos que no son tomados en cuenta en este planteamiento.

Existe una tendencia de clasificar las conductas de los niños y de diagnosticarlas en búsqueda de tratamientos "objetivos". En este sentido se omite aquella información que apunte a cualquier otra información que hable de cada sujeto y distraiga la atención de lo preestablecido en el DSM. Vasen (2011) menciona que la clasificación se funda en una selección de datos, la cual deriva en la construcción de un dato que es un vínculo unívoco entre un hecho y su significado. Esta objetivación recae sobre las infancias, las cuales pretenden ser abordadas de manera tecnocrática, autoritaria y mercantilista.

El riesgo de no abordar a los alumnos desde su subjetividad, tiene como consecuencia el hecho de pensar que las historias familiares no repercuten en las conductas de los menores, y que todos están hechos por el mismo molde; por esta razón se les aplican los mismos cuestionarios para diagnosticar a los niños con características similares. Untoiglich (2013) propone que los profesionales de la salud tendríamos que utilizar un lápiz para escribir las primeras hipótesis diagnósticas, las cuales no deberían constituirse nunca en una marca indeleble en la vida de un sujeto, debido a que un niño se encuentra en proceso de constitución de su subjetividad.

En el ámbito escolar de la ciudad de Guadalajara se ha "medicalizado" el abordaje del TDAH, incrementándose cada vez más la canalización al paidopsiquiatra o al neurólogo, de los alumnos que presentan conductas tales como desatención, falta de organización y de sistematización al momento de seguir instrucciones, o movimiento excesivo, entre otras, para ser "atendidos" con psicofármacos.

La escala más utilizada para canalizar a los niños al neurólogo, es la creada en 1969 por C. Keith Conners (anexo 1), la cual consiste en 48 ítems referentes a actividades en niños que podrían estar relacionadas con la falta de atención o la hiperactividad y se califican a partir de un rango de categorías como: "mucho", "bastante", "un poco" o "para nada". Estas preguntas se evalúan en el contexto escolar a partir del criterio del docente, y en el familiar, los padres de familia valoran que tanto su hijo es inquieto o distraído.

Para evaluar la atención ésta no se visualiza como una función, sino como una conducta. Los ítems utilizados son: "Se distrae fácilmente, tiene escasa atención", lo cual abriría el campo para más opciones acerca de la explicación de la distracción, ante la aclaración que es por falta de la 
atención. Otro de los ítems es: "No termina las tareas que empieza", esta conducta, también podría abarcar diversas explicaciones y abordajes que no aterrizarían únicamente en una deficiencia en la atención, sino que podrían ser resultado desde una falta de identificación con la actividad realizada, hasta fallas en la estructuración psíquica.

Untoiglich (2007) profundiza en los orígenes de este cuestionario, y menciona que no fue confeccionada en su origen para realizar un diagnóstico específico de TDAH, sino como un proceso de screening ${ }^{1}$, para identificar las dificultades conductuales en los niños. El objetivo era la detección no el realizar un diagnóstico a partir de este instrumento; sin embargo, su rápida aplicación ha cambiado su función en los ambientes escolares, ya que los resultados, en ocasiones, condicionan a los padres a "atender" a su hijo llevándolo a un neuropediatra. No obstante, en la práctica, en la Dirección de Psicopedagogía este instrumento se convierte en el diagnóstico, ya que en casi la totalidad de los casos, al acudir a las instancias de salud públicas, por la falta de tiempo y presupuesto para análisis clínicos, en citas de quince minutos se confirma el diagnóstico y se les prescribe en la mayoría de los casos Metilfenidato, que es el medicamento más usado en el Trastorno de Déficit de Atención.

Es interesante advertir cómo socialmente las farmacéuticas han enviado el mensaje con ayuda de los medios de comunicación y congresos médicos, en los que promueven que los efectos secundarios son casi nulos. Según la Facultad de Medicina de la UNAM, estas son las reacciones secundarias y adversas:

Los eventos adversos más comúnmente reportados $\left({ }^{3} 10 \%\right)$ incluyen: Cefalea, dolor estomacal, pérdida del apetito, insomnio. Otros eventos adversos comunes $\left({ }^{3} 1 \%\right.$ a $\left.<10 \%\right)$ incluyen: Reacción agravada, astenia, hipertensión, náusea y/o vómito, dispepsia, pérdida de peso, tics, mareo, labilidad emocional, somnolencia, ansiedad, depresión, nerviosismo, hostilidad, exantema. Eventos adversos poco comunes $\left({ }^{3} 0.1 \% \mathrm{y}<1 \%\right)$ incluyen: dolor torácico, fiebre, lesiones accidentales, malacia, dolor, intentos suicidas, migraña, taquicardia, diarrea, incontinencia fecal, incremento del apetito, calambres en las piernas, apatía, pensamientos anormales, sueños anormales, alucinaciones, confusión, hipercinesia, trastornos del sueño, trastornos del lenguaje, vértigo, tos aumentada, epistaxis, alopecia, prurito, urticaria, diplopía, polaquiuria, hematuria y urgencia urinaria (Metilfenidato (s.f.)).

Las estadísticas de riesgo, en relación a los efectos secundarios, según este artículo es menor a 10 por ciento de los sujetos que consumen el medicamento, porcentaje que éticamente se les debería comunicar a los padres de familia, quiénes son los responsables de tener la última palabra al respecto. En otro artículo del periódico La Jornada, titulado: "Ritalín: más de 50 años en el mercado

${ }^{1}$ Según la doctora Untoiglich (2007: 129), esta palabra podría traducirse como filtrado, pasar por el tamiz; así como también, bloquear la visión, opacar o censurar. 
farmacéutico y sigue causando polémica", refieren que "La literatura médica tiene reportadas una decena de muertes relacionadas con el Ritalín" (Norandi, 2006), pero según la doctora Matilde Ruiz, jefa de neurología del Instituto Nacional de Pediatría, esto se debe a que la mayoría de esos pacientes tenían una cardiopatía previa, por lo que recomienda que antes de medicar se realice una evaluación cardiovascular. Esta aseveración, deja entrever que el medicamento también tiene otro tipo de efectos secundarios, los cuales también deberían ser especificados.

Es importante rescatar que el artículo referido, además abordaba el debate del Ritalín, ya que en 2006 comenzó la discusión entre los legisladores acerca del diagnóstico y uso de este medicamento. Como resultado, en 2009 se hicieron modificaciones a la Ley de Educación en el Diario de la Federación, en la cual en el artículo 75 refieren que los que prestan servicios educativos no pueden administrarle a los educandos, sin prescripción médica o autorización de los padres, sustancias psicotrópicas o estupefacientes ${ }^{2}$, ni promover su uso o negar el servicio a niños y adolescentes que presenten problemas de aprendizaje y se condicione su permanencia en el plantel, si se somete a tratamientos médicos específicos.

Extrañamente estas modificaciones de ley no tuvieron impacto, ya que su difusión a nivel nacional fue casi nula y en la práctica los condicionamientos a los alumnos que deciden no visitar a un neurólogo, siguen existiendo por el desconocimiento de este pronunciamiento. La tendencia hasta la fecha ha sido, el culpabilizar y "pasarse la bolita", entre docentes, padres de familia y psicólogos, sin lograr un consenso. En la Dirección de Psicopedagogía, la visión es que "no les toca" hacer el diagnóstico, por lo que el procedimiento es aplicar el Conners y el DSM-IV para "descartar" el TDAH en todos los casos que son canalizados. Al no ser "tocados" por esta situación, la problemática de sobrediagnosticación antes mencionada continúa.

Fabiola Nieto (s/f), coincide con esta percepción en su artículo "Ritalín, negocio en auge" en el cual refiere:

Dado el escaso control de diagnóstico y la falta de preparación de los psicólogos de primer contacto en esta área, se ha registrado un indiscriminado aumento en la prescripción de las drogas para el control del TDHA llamadas Ritalin (metilfenidato) y Stratera (atomoxetina), este último contando con riesgo potencial de daño hepático grave, según advertencias de la Food and Drug Administration (FDA).

Mientras los psicólogos educativos, no se concienticen de esta situación, seguirán recibiendo diagnósticos menores que presentan estas problemáticas, pero que pudieran apuntar a problemáticas familiares, bullying, depresión, psicosis o abuso sexual y que la única forma de saberlo sería escuchando a los menores y dando tiempo a los procesos y a trabajar de forma conjunta para poder tener diagnósticos diferenciales más acertados.

\footnotetext{
${ }^{2}$ Cabe aclarar que el metilfenidato está considerado como un estupefaciente en el capítulo quinto, artículo 234, de la Ley Federal de Salud.
} 
Punta, M (2007: 52-53) comenta que se confunde el ADD con otras patologías como la ansiedad, extremadamente frecuente por la alta proporción de procesos fóbicos tempranos que evolucionan mal, el trastorno narcisista no psicótico, estados depresivos crónicos combinados o no con ansiedad, patología psicótica, interferencias de la actividad mental, causadas por la emergencia de ideas obsesivas incontrolables que le impiden al niño atender lo que está haciendo, patologías del medio no reconocidas como tales, que frustran al niño en todos sus procesos vitales o patologías de la agresión reactiva.

En este sentido ¿qué cambios se podrían hacer al interior de la Dirección de Psicopedagogía? En primera instancia, sería trascendental que se cambiaran los abordajes de los alumnos, a partir del conocimiento de estas nuevas subjetividades, para atender al llamado de los síntomas de estos niños. Escucharlos desde su propia historia, sin imponerles todo un discurso "científico" que no les permite moverse de su posición de "enfermos" o de "niños problema".

En este orden de ideas, Silvia Bleichmar (2008) plantea la necesidad de hablar sobre herramientas que permitan pensar los problemas cotidianos y que atiendan al profundo sentimiento de orfandad de posibles estrategias de solución, con las que cuentan los docentes para enfrentar nuevas situaciones, ya que existen cambios en los procesos de subjetivación, que desencadenan nuevos modos de conducta en los que algunos productos fantaseados o deseos reprimidos se han llevado a la realidad, en las nuevas manifestaciones generales de violencia. Asimismo, hay que tener en cuenta los nuevos métodos de enlace en los que se establecen nexos de ensamblaje que reemplazan las antiguas formas familiares de agrupamiento. Todas estas novedades, causan el "traumatismo de los docentes, de los psicólogos y los psicopedagogos ante las nuevos interrogantes que plantean los chicos y de cómo encarar estas nuevas problemáticas" (Bleichmar, 2008: 126).

Gisela Untoiglich (2007) menciona que la atención por sí sola no es el factor principal para que los chicos no tengan éxito en la escuela, sino que se necesitan requerimientos psíquicos y académicos necesarios para que un niño pueda ingresar a la educación primaria. Al respecto, señala que

difícilmente podrá atender los requerimientos escolares un niño que no tenga mínimamente tramitadas cuestiones ligadas a su origen, a su historia familiar, al lugar que ocupa en el deseo de sus padres; que tenga ciertos diques construidos, límites incorporados, posibilidades sublimatorias de aplazamiento de la satisfacción inmediata y que pueda renunciar a la presencia real e inmediata de la madre en pos de la satisfacción de recibir más tarde la felicitación de mamá cuando vea las producciones escolares. Académicamente se espera que tenga ciertas nociones de lectura y escritura, e igualmente de matemática; que identifique el momento de juego únicamente limitado para el recreo, y que se considere al programa como una ruta fija por la cual se debe transitar, prestando atención a contenidos que, por momentos, son lejanos a los intereses cotidianos de los niños (ibídem: 128). 
¿Pero cómo lograr que estos requerimientos psíquicos y académicos sean cubiertos? Es importante que se cuente con un mayor número de profesionales de la salud, que puedan estar al tanto de estos procesos en los niños, pero que estén lo suficientemente capacitados para orientar a los alumnos y a los docentes en el respeto a dichos procesos, a fin de que pueda existir un seguimiento y un apoyo desde la escuela, sin excluir a los alumnos de la misma.

El psicoanálisis propone formas de abordaje en las que el déficit de atención se convierte en un síntoma en búsqueda de una interpretación; es decir que no se trata de erradicar "violentamente" la conducta no deseada, sino de encontrar las causas inconscientes.

Siguiendo este orden de ideas, Vasen (2007) refiere que las intervenciones psicoanalíticas inciden sobre los síntomas a través de lo que nos representamos como sus circuitos productores, en general fantasmáticos. Intentando deconstruirlos, no suprimirlos. "Tampoco restituir el mismo equilibrio perdido pues en él, anidan las líneas de fractura que llevaron al despliegue o repliegue sintomal. La resultante de las intervenciones será un nuevo equilibrio" (ibídem: 124).

En el caso del trastorno de déficit de atención, puede ser una forma en la que los niños "demandan" ayuda o como mencionan Sanfeliu (2011) y Janin (2007) sin estos síntomas los niños "no existirían", es una lucha por intentar demostrar que están vivos ya que a partir del despliegue motriz, los otros les corroboran que existen.

Si el psicoanálisis apuesta por la interpretación de los síntomas, el método de abordaje infantil, según Pundik (2006), radica en escuchar a los padres con el fin de conocer su versión acerca de la problemática de su hijo, con el propósito de intentar ordenar las hipótesis acerca de las circunstancias que puedan haberse hecho presentes en el momento del desencadenamiento de los síntomas. Posteriormente escuchan el discurso del niño, sin privilegiar las pautas previamente establecidas para todos los casos; es decir, que no se trata de encontrar una concordancia con algún diagnóstico previo, ya que intentan sostener la singularidad del sujeto.

Susana Perales (2011: 61) profundiza en el tema y refiere que en la clínica psicoanalítica "es la singularidad, la que se anuda a la voz del sufriente en los momentos de crisis... cuando el discurso no le alcanza al sujeto para entender lo que le pasa en su cuerpo o en sus pensamientos". Las particularidades del caso, dan orientación del diagnóstico, la construcción clínica y la dirección de la cura. Cuando acude una familia a un analista, éste se centra en el discurso de la misma para poder intervenir, sin quedarse únicamente en la observación de las conductas, ya que a partir de las características específicas de esa familia, se podrá profundizar en su sufrimiento psíquico y eventualmente el síntoma se transformará; de ahí surge el título de Gisela Untoiglich (2013) En la infancia los diagnósticos se escriben con lápiz, metaforizando el hecho de que son una guía en la intervención clínica, mas no una etiqueta fija.

Lo que esta autora plantea es que el psicoanálisis puede demostrar que la atención "no es por sí misma un fenómeno cuantitativo, sino que es una perturbación de la atención por otras exigencias de satisfacción, es una función estrictamente ligada a los modos de satisfacción de un sujeto". 
Interpreta que los niños se distraen por los significantes que marcan su historia y lo sustraen de las tareas educativas y familiares habituales, haciéndole vagabundear en sus fantasías, dejándolo cautivo de la preeminencia de las relaciones imaginarias con los otros, o transformándolo en la presa de un cuerpo inquieto que lo desborda, como una "hiperactividad" desenfrenada (ibídem: 64).

Para Janin, (2007) el déficit de atención implicará tanto un déficit en la constitución adentroafuera, si el niño está inmerso en un mundo en el que los estímulos no pueden ser diferenciados, con la libidinización cuando lo que falla es la constitución de dirigirse al mundo, la constitución narcisista del yo cuando no puede salirse de sí; pero también puede haber un retraimiento secundario por depresión, o una dificultad para acotar la fantasía o un estado de alerta producto de situaciones de violencia, entre otras posibilidades (ibídem: 58 ).

Entonces el Trastorno de Déficit de Atención que desde la óptica del Manual Diagnóstico de Trastornos Mentales puede ser visto como uno solo, constituido por ciertas conductas, bajo la lupa psicoanalítica, podría apuntar a síntomas que podrían estar vinculados con diversas dificultades que el niño presenta.

Es interesante como Janin (2007), hace un análisis de las causas más comunes que ha identificado en su práctica clínica:

- El niño "dormido", niños que sin ser autistas tienen momentos autistas. Están como dormidos, recluidos en una especie de vacío.

- El niño que confunde adentro-afuera, no diferencia entre estímulo y pulsión. Al no diferenciar los estímulos externos de los internos, reacciona de igual modo a ambos. En estos niños predomina un funcionamiento por urgencias, con captación de frecuencias y ritmos, internos y externos. Tienden a saltar de un extremo al otro del aula, sin metas claras.

- El niño "objeto" a mostrar, en estos niños predomina el deseo de ser mirados y no pueden fijar la mirada en otro. La atención está dirigida a preservar-se en un lugar en el que confluyen esas miradas, pero no pueden registrar sucesos ni palabras.

- "No escucho porque ya lo sé", representa los trastornos en la constitución de la investidura de atención por desmentida de la castración (por no soportar una fractura narcisista). No hay tolerancia a las heridas narcisistas, sólo atienden a lo que les resulta fácil.

- “¿Me quiere o no me quiere?”, se refiere a los niños que han constituido las investiduras de atención en relación con los intercambios afectivos pero no en relación con el conocimiento. Buscan la aprobación afectiva, el cariño de los maestros, pero no pueden escucharlos. Niños en los que el narcisismo, como amor a sí mismo, se sigue sosteniendo en la mirada amorosa de otro. Si ésta falta, no pueden encontrarse a sí mismos y sólo pueden buscarse en la conexión afectiva con otro.

- El niño triste. Constitución de las investiduras de atención y posterior retracción por duelo.

- El niño que juega. En estos casos los niños son desatentos por retracción a un mundo 
fantasmático. Otra de las posibilidades es que el niño haya retirado sus investiduras del mundo para investir sus fantasías. Son niños que sueñan despiertos, juegan en clase y es como si se mantuvieran en un mundo paralelo.

- El niño que está en "alerta" permanente. Estos niños sufren de una sobre atención primaria, aunque son catalogados como desatentos. No pueden centrar la atención en ningún elemento, sino que pasan de uno a otro, en un zapping permanente. Están conectados con el afuera pero lo identifican como peligroso.

- El niño de pensamiento "confuso". No pueden organizar sus pensamientos y confunden deseos y realidad. Manifiestan una gran labilidad en su acercamiento al mundo, en sus vínculos y en su modo de expresar sus afectos (ibídem: 58-67).

Dentro de estas categorías clínicas, se encuentran en juego en algunos casos el proceso psíquico primario, en donde no existe una diferencia entre representación y pulsión en otros los secundarios, cuando el yo posee una reserva de investiduras que le permiten efectuar la inhibición.

A partir de estos planteamientos, Janin (2007), refiere que para poder atender sostenidamente a la palabra de los maestros es necesario el funcionamiento de los dos procesos. En el caso del secundario, si no se puede efectuar la inhibición, cada palabra, gesto o movimiento desencadenará una sucesión de asociaciones imparables. En caso de que no opere el proceso primario, existe el riesgo de que la palabra del maestro no pueda ser valorizada o que el niño se quede atrapado en sus registros olfativos.

Gisela Untoiglich (2011: 43) propone que la atención desde la perspectiva psicoanalítica, es "una función ligada al Yo que se va constituyendo en un proceso histórico intersubjetivo. Es en el encuentro con el Otro que se va a delimitar, a qué y cómo se atiende".

Esta visión de la atención como proceso, pone énfasis en el lugar y la importancia que tienen los adultos en el niño, porque no es únicamente esta función la que se constituye, sino sería importante no perder de vista que tanto niños como adolescentes se encuentran en formación y sus síntomas se vinculan también con el contexto y las interacciones que tengan con quienes vivan. Asimismo, si los adultos que están a su alrededor, no fungen como sostén, el menor sentirá que se encuentra a la deriva.

El gran riesgo en el que se encuentran los niños y adolescentes, es que como adultos nos desresponsabilicemos de su sufrimiento. Pero parecería que es el objetivo de los abordajes médicos que dejan a un lado la subjetividad de los menores, apuntando a diagnósticos al vapor que "resuelven" de forma temporal problemáticas más complejas. Es desde las políticas públicas, que sería necesario crear dispositivos de prevención de patología grave en la infancia, pero no a partir de repartir diagnósticos de forma masiva, sino desde la escucha y la intervención analítica, para evitar que los niños que presentan estos síntomas sean excluidos del sistema escolar y queden expuestos a riesgos más altos. 
En el caso de la Dirección de Psicopedagogía, así como en otras instituciones de asistencia pública en esta ciudad, sería importante que cambiaran de inicio la visión estadística por la de calidad de atención. La tendencia es a engordar las cifras, como si se tratara de productividad en masa. Cada niño tiene una historia, una familia y la gravedad de sus problemáticas también difiere, por lo tanto, el trabajo tendría que planearse partiendo de esa demanda específica.

Todavía existen muchos caminos por recorrer, no obstante, el plano humano de trabajo colaborativo no se puede seguir dejando de lado, según mi opinión. Ésta puede ser una oportunidad de cambio y de transformación en el sistema escolar mexicano, pero sólo el trabajo coordinado y bajo un proyecto que tome en cuenta las reflexiones expresadas anteriormente, harán posible alcanzar resultados positivos, pero el enfoque en la Dirección de Psicopedagogía tiene que cambiar, ya que si la prioridad continúa apoyándose en las estadísticas y los resultados rápidos, se seguirán ignorando las problemáticas reales de los alumnos, y habrá cada vez menos espacios para ser escuchados. Asimismo, los docentes necesitan también espacios para ser abordados, no desde un enjuiciamiento o desde una serie de estrategias preestablecidas, sino que en ocasiones desde su propia práctica profesional e historia personal a fin de tener resultados más significativos.

\section{Bibliografía}

Bange, F. (2010). Hiperactivité: Des enfants “Tornades”? Les Grands Dossiers des Sciences Humaines, (20), pp. 56-57.

Bleichmar, S. (2008). Subjetividad en riesgo: herramientas para su rescate. En: S. Bleichmar. Violencia social-Violencia escolar. De la puesta de límites a la construcción de legalidad (pp. 123-140). Buenos Aires: Noveduc.

Colín, A. (2001). La historia familiar, la subjetividad y la escuela. En Hermosillo, T. El traspatio escolar. Una mirada al aula desde el sujeto (p. 135). México: Paidós.

Decreto por el que se adicionan las fracciones XIII, XIV y XV al artículo 75 y una fracción III al artículo 76 de la Ley General de Educación (2009). Diario Oficial de la Federación. México: 17 de abril de 2009. Recuperado el 2 de febrero de 2013 desde:http://dof.gob.mx/nota_detalle.ph $\mathrm{p}$ ? codigo $=5087520 \&$ fecha $=17 / 04 / 2009 \&$ print $=$ true

Dubrovsky, S. (1998). "Aportes de la teoría histórico-cultural de Vigotsky al trabajo en el aula y en la clínica". En: Los atrasados escolares. El trabajo en el aula y en la clínica. Buenos Aires: Noveduc, pp. 6-13.

Dueñas, G. (Abril 2007). ¿Nuevos dispositivos de control de la conducta? En: La atención y el aprendizaje. Niños inquietos. ¿Actividad o hiperactividad? Buenos Aires: Noveduc, pp. 3437.

Janin, B. (2007). Un niño que se mueve demasiado. En Janin, B. Niños desatentos e hiperactivos $A D D / A D H D$ (pp. 73-96). Buenos Aires: Noveduc 
Metilfenidato (s/f). Recuperado el 18 de septiembre del 2013, desde_http://www.facmed.unam.mx/ bmnd/gi_2k8/prods/PRODS/Metilfenidato.htm

Nieto, Fabiola (s/f) "Ritalín. Déficit de atención, negocio en auge". Revista Fortuna, negocios y finanzas [en línea]. Recuperado el 7 de febrero de 2013, desde http://www.revistafortuna.com. $\mathrm{mx} /$ opciones/archivo/2005/junio/html/salud/ritalin.htm

Norandi, M. (5 de septiembre, 2006) Ritalín: más de 50 años en el mercado farmacéutico y sigue causando polémica. La Jornada [en línea]. Recuperado el 3 de septiembre de 2013, desde http://www.jornada.unam.mx/2006/09/05/index.php?section=sociedad\&article=040n1soc

Perales, S. (2011). Los síntomas contemporáneos y el psicoanálisis. En Gerez, M. Desafíos en la clínica psicoanalitica actual (pp. 59-65). México: Círculo Psicoanalítico Mexicano A.C.

Pundik, J. (2006). El niño hiperactivo, déficit de atención y fracaso escolar. Guía para padres y docentes. Madrid: Filium.

Punta, M. (2007). El ADD/ADHD como caso testigo de la patologización de la diferencia. En Benasayag, L. ADDH. Niños con déficit de atención e hiperactividad ¿Una patología de mercado? (pp. 47-70). Buenos Aires: Noveduc.

Sanfeliu, I. (2011). La hiperactividad. La acción inagotable. Madrid: Biblioteca nueva.

Untoiglich, G. (2007). Intersecciones entra la cínica y la escuela. En Janin, B. Niños desatentos e hiperactivos ADD/ADHD. Reflexiones críticas acerca del Trastorno por Déficit de Atención con o sin Hiperactividad (pp. 125-145). Buenos Aires: Noveduc.

— (2011). Versiones actuales del sufrimiento infantil. Una investigación psicoanalítica acerca de la desatención y la hiperactividad. Buenos Aires: Noveduc.

— (2013). En la infancia los diagnósticos se escriben con lápiz. La patologización de las diferencias en la clínica y la educación. Buenos Aires: Noveduc.

Vasen, J. (2007). La atención que no se presta: El mal llamado TDAH. Buenos Aires: Noveduc.

- (2008). Las certezas perdidas. Buenos Aires: Paidós.

— (2011). Una nueva epidemia de nombres impropios. El DSM-V invade la infancia en la clínica y las aulas. Buenos Aires: Noveduc.

Recibido: 14/10/13

Dictaminado: 11/02/14

Corregido: 20/02/14

Aceptado: 06/03/14 


\section{Anexo I \\ Cuestionario de conducta de CONNERS para el diagnóstico de TDA CUESTIONARIO DE CONDUCTA EN EL HOGAR \\ (C.C.I.; Parent`s Questionnaire, C. Keith Conners). Forma abreviada. \\ Utilizar este cuestionario para obtener una descripción de las conductas de los alumnos. \\ Los datos obtenidos deben trasladarse a la tabla diagnóstica del DSM-IV}

\section{Descriptores}

1. Se manosea los dedos, uñas, pelo, ropa.

2. Trata irrespetuosamente a personas mayores

3. Tiene dificultad para hacer o mantener amistades.

4. Es impulsivo, irritable.

5. Quiere controlar y dirigir en cualquier situación.

6. Se chupa el dedo, la ropa o las mantas.

7. Es llorón.

8. $\quad$ Es desgarbado en su porte externo.

9. Está en las nubes, ensimismado.

10. Tiene dificultad para aprender.

11. Es más movido de lo normal.

12. Es miedoso.

13. No puede estarse quieto.

14. Es destructor (ropas, juguetes, otros objetos).

15. Es mentiroso.

16. Es retraído, tímido.

17. Causa más problemas que otro de su misma edad.

18. Su lenguaje es inmaduro para su edad.

19. Niega sus errores o echa la culpa a otros.

20. Es discutidor.

21. Es huraño, coge berrinches.

22. Roba cosas o dinero en casa o fuera.

23. Es desobediente, obedece con desgana.

24. Le preocupa excesivamente estar solo, la enfermedad.

25. No acaba las cosas que empieza. 
CUESTIONARIO DE CONDUCTA EN EL HOGAR (continuación)

(C.C.I.; Parent`s Questionnaire, C. Keith Conners). Forma abreviada.

\section{Descriptores}

26. Es susceptible, se "pica" fácilmente.

27. Tiende a dominar, es un "matón".

28. Hace movimientos repetitivos durante ratos.

29. Es a veces cruel con los animales o niños pequeños.

30. Pide ayuda y seguridad como si fuese más pequeño.

31. Se distrae fácilmente, escasa atención.

32. Le duele la cabeza frecuentemente.

33. Cambia bruscamente sus estados de ánimo.

34. No acepta restricciones o reglamentos, desobediente.

35. Se pelea con mucha frecuencia por cualquier motivo

36. No se lleva bien con sus hermanos.

37. Sus esfuerzos se frustran fácilmente, es inconstante.

38. Suele molestar frecuentemente a otros niños.

39. Habitualmente es un niño triste.

40. Tiene poco apetito, caprichoso con las comidas.

41. Se queja de dolores de vientre.

42. Tiene trastornos de sueño.

43. Tiene otros tipos de dolores.

44. Tiene vómitos con cierta frecuencia.

45. Se siente marginado o engañado en su familia.

46. Suele ser exagerado, "fardón".

47. Deja que le manipulen o abusen de él.

48. No controla bien el pis o tiene dificultades de defecación. 


\section{CUESTIONARIO DE CONDUCTA EN LA ESCUELA}

(C.C.E.; Teacher`s Questionnaire, C. Keith Conners). Forma abreviada.

Utilizar este cuestionario para obtener una descripción de las conductas de los alumnos. Los datos obtenidos deben trasladarse a la tabla diagnóstica del DSM-IV

\section{Descriptores}

1. Tiene excesiva inquietud motora.

2. Emite sonidos molestos en situaciones inapropiadas.

3. Exige inmediata satisfacción de sus demandas

4. Se comporta con arrogancia, es irrespetuoso.

5. Tiene explosiones impredecibles de mal genio.

6. Es susceptible, demasiado sensible a la crítica.

7. Se distrae fácilmente, escasa atención.

8. Molesta frecuentemente a otros niños.

9. Está en las nubes, ensimismado.

10. Tiene aspecto enfadado, huraño.

11. Cambia bruscamente sus estados de ánimo.

12. Discute y pelea por cualquier cosa.

13. Tiene actitud tímida y sumisa ante los adultos.

14. Intranquilo, siempre en movimiento.

15. Es impulsivo e irritable.

16. Exige excesivas atenciones del profesor.

17. Es mal aceptado en el grupo.

18. Se deja dirigir por otros niños.

19. No tiene sentido de las reglas del "juego limpio".

20. Carece de aptitudes para el liderazgo.

21. No termina las tareas que empieza.

22. Su conducta es inmadura para su edad.

23. Niega sus errores o culpa a los demás.

24. No se lleva bien con la mayoría de sus compañeros.

25. Tiene dificultad para las actividades cooperativas.

26. Sus esfuerzos se frustran fácilmente, es inconstante.

27. Acepta mal las indicaciones del profesor.

28. Tiene dificultades de aprendizaje escolar. 